\title{
Thesen zur handlungstheoretischen Neuorientierung sozialgeographischer Forschung
}

In der sozialgeographischen Theoriediskussion besteht ein weitreichender Konsens darüber, da $B$ im Rahmen dieser Disziplin nur dann eine relevante Forschung betrieben werden kann, wenn es gelingt, sie auf explizite, kontrollierte und logisch konsistente Weise in allgemeinere sozialwissenschaftliche Theorien zu integrieren. Bei den bisherigen Versuchen, die sozialwissenschaftlichen Leitlinien auf die sozialgeographische Fragestellung hin abzuwandeln und zu erweitern, wurde aber offensichtlich, da $B$ dieser Anspruch tiefgreifende Probleme aufwirft. Führende Fachvertreter haben deshalb den Versuch unternommen, die auftretenden Schwierigkeiten unter Rückgriff auf wissenschaftstheoretische Argumente zu überwinden. Zwei der zur Zeit wichtigsten Forschungsansätze der Sozialgeographie sind das Ergebnis derartiger Bestrebungen: Der sogenannte «spatial approach» angelsächsischer Tradition, der in modifizierter Form von Dietrich BARTELS in die deutsche Sozialgeographie eingebracht wurde, und die sogenannte «humanistic geography», die u. a. von ANNE BUTTIMER vorgeschlagen wird.

Der Grundgedanke der folgenden Ausführungen (1) lautet, daß beide Bezugnahmen auf wissenschaftstheoretische und sozialwissenschaftliche Leitbilder inkonsistent sind. Diese Inkonsistenzen werden zudem als wichtiger Grund für die weiterhin geringe praktische Relevanz sozialgeographischer Forschungsergebnisse und für die andauernde Isolation der Sozialgeographie innerhalb der sozialwissenschaftlichen Disziplinen betrachtet. Zur Beseitigung dieser Mängel möchte ich den Vorschlag einer handlungstheoretischen Neuorientierung sozialgeographischer Forschung unterbreiten, der sich begrifflich und logisch konsistent auf seine wissenschaftstheoretischen und sozialwissenschaftlichen Grundlagen bezieht und letztere kontrolliert erweitern soll. Damit greife ich die erste und bisher wirkungsvollste, in mehrerer Hinsicht aber unbefriedigende Anregung von D. BARTELS $(1968 ; 1970)$ auf und stelle sie in einen neuen Argumentationszusammenhang (2).

Diese Argumentation wird anhand von sechs Thesen vorgestellt, deren Erläuterung das Vorhaben transparent machen soll. Zu dessen Verwirklichung erarbeite ich zusätzlich einige Orientierungshilfen. Zuerst ist aber die aktuelle Ausgangssituation ge- nauer zu charakterisieren, damit die problematischen Implikationen des raumwissenschaftlichen Forschungsprogramms («spatial approach») und seiner humanistischen Alternative phänomenologischer Prägung differenzierter behandelt werden können.

\section{Sozialgeographie zwischen Raumwissenschaft und Phänomenologie}

Das Hauptziel raumwissenschaftlich orientierter Geographen besteht bekanntlich in der Aufdeckung von sogenannten Raumgesetzen (vgl. BUNGE, 1966; BARTELS, 1968) mittels der choristisch-chorologischen Methodik. BARTELS übernimmt die allgemeine Wegleitung dieses Programms und erweitert es für die Sozialgeographie in zweifacher Hinsicht. Unter Berufung auf $K$. R. POPPER u. a. fordert er (1968, 45ff.; 1970, 37), daß die entdeckten Gesetze zu einer chorologischen Theorie systematisiert werden. Aus ihr sollen dann Kausalerklärungen des erdräumlichen Gesamtmusters einer Gesellschaft sowie Technologien und Prognosen für die Raumplanung deduktiv abgeleitet werden (vgl. 1968, 52ff., 1975, 47). Die zweite Erweiterung betrifft die Anwendung der formalen raumwissenschaftlichen Modelle auf die «erdoberflächlichen Verbreitungs- und Verknüpfungsmuster im Bereich menschlicher Handlungen und ihre Motivationskreise» (1970, 30). Damit beabsichtigt er (vgl. 1970, 33ff.), in letzter Konsequenz, die Erweiterung der Handlungstheorien von M. WEBER. V. PARETO und T. PARSONS durch die Berücksichtigung der erdräumlichen Verteilung der Bedingungen, Mittel und Folgen des Handelns und die Anwendung der raumwissenschaftlichen Methodik auf die "Problemzusammenhänge dieser sozialwissenschaftlichen Grundperspektive» (BARTELS, 1970, 33). So definiert BARTELS die Sozialgeographie als handlungsorientierte Raumwissenschaft, deren Zielsetzung und Metho-

Benno Werlen, Geographisches Institut der Universität Zürich-Irchel, Winterthurerstr. 190, 8057 Zürich 
dologie er aus der kritisch-rationalen Wissenschaftstheorie von K. R. POPPER und der raumwissenschaftlichen Tradition der Geographie ableitet.

Für die Vertreter der «humanistic geography» stellt der «spatial approach» eine technokratische Forschungskonzeption dar, die durch die humanistische Alternative überwunden werden sollte (vgl. LEY, 1981, 253f.). Ihre Forderung lautet, daß nicht die $o b$ jektive Erklärung der choristischen Verteilungsmuster verschiedener Gegebenheiten die Aufgabe der Sozialgeographie sein solle, sondern das subjektive Verstehen (vgl. BUTTIMER, 1976) der Verhaltensweisen «konkreter Menschen» in ihrem erdräumlichen Kontext. Dazu sei eine Sozialgeographie nach dem Leitbild der verhaltenstheoretischen Wissenschaften aufzubauen. Die Abwandlung und Erweiterung der Verhaltenstheorie wird durch die besondere $\mathrm{Be}$ rücksichtigung der erdräumlichen Differenzierung der verhaltenssteuernden Informationen angestrebt: einerseits durch die Integration der subjektiven «mental map» in das Verhaltensmodell und andererseits durch die Erforschung der verhaltensleitenden Bedeutung der Sinngehalte von Orten, die als «meaning of places» thematisiert werden (vgl. TUAN, 1976, 286f.; BUTTIMER, 1976, 283ff.). Zielsetzung und Methodologie dieser humanistischen Forschungsperspektive (vgl. LEY, 1981, 253) werden mit der phänomenologischen Wissenschaftstheorie von E. HUSSERL und A. SCHÜTZ begründet (vgl. BUTTIMER, 1976, 279ff.; 1979, 245ff.).

Aufgrund der beiden Argumentationsstränge kann man - wie das für die aktuelle Theoriediskussion kennzeichnend ist - zur Auffassung gelangen, da $B$ aus dem Kritischen Rationalismus nur eine sozialgeographische Raumwissenschaft im «Vorfeld soziologischer Handlungstheorie» (BARTELS, 1968, 165) abgeleitet werden kann, die mit der phänomenologischen Alternative deshalb nicht zu vereinbaren ist, weil letztere sich auf das subjektive Verstehen derVerhaltensweisen konkreter Menschen konzentrieren will. Im Gegensatz dazu möchte ich hier argumentieren, da $\beta$ Verhaltens- und Handlungstheorie zwar nicht gleichgesetzt werden dürfen, die beiden Metatheorien von POPPER und SCHÜTZ hingegen die Grundlage zur Entwicklung einer handlungstheoretischen Sozialgeographie abgeben. Die weiteren Thesen betreffen das Verhältnis zu den sozialwissenschaftlichen Bezugstheorien sowie deren Abwandlung und Erweiterung im Hinblick auf die sozialgeographische Fragestellung.

\section{Von der verhaltenstheoretischen zur handlungs- theoretischen Sozialgeographie}

Die erste These betrifft das Verhältnis zwischen der von BARTELS angesprochenen Handlungstheorie und der von BUTTIMER vorgeschlagenen Verhaltenstheorie. Zudem geht es um die Überprüfung der
Leistungsfähigkeit dieser beiden Theorien im Hinblick auf die Erreichung des sozialgeographischen Forschungsziels, eine Gesellschaftsanalyse unter Berücksichtigung der erdräumlichen Dimension zu realisieren.

\section{These 1: Wenn die Sozialgeographie ihr Forschungs- ziel erreichen will, dann sollte sie nicht verhal- tenstheoretisch entworfen werden, sondern hand- lungstheoretisch.}

Bevor diese These begründet werden kann, ist auf die Unterschiede zwischen verhaltens- und handlungstheoretischer Forschungslogik aufmerksam zu machen.

Die in Abb. 1 rekonstruierte Argumentationsstruktur zeigt, da $B$ in der kognitiven psychologischen Verhaltenstheorie die menschlichen Tätigkeiten zwar nicht mehr - wie im klassischen Behaviorismus - als unmittelbar von beobachtbaren Sinneseindrücken bewirkt aufgefaßt werden (vgl. GOLD, 1980, 42). Die Grundstruktur der Erklärung bleibt aber - auch in der sozialgeographischen Abwandlung - dieselbe: Tätigkeiten werden als Reaktionen auf Umweltinformationen begriffen, allerdings in der Form, wie sie über kognitive $Z$ wischenprozesse verarbeitet verhaltenswirksam werden. Persönlichkeitsmerkmale und die beim Individuum repräsentierten sozial-kulturellen Faktoren führen demgemäß zu einer bestimmten Ausprägung der Motive und Bedürfnisse sowie dem manifesten Anspruchsniveau. In dieser Form werden letztere zu den kognitiv wahrnehmungs- und verhaltensleitenden Faktoren. Von ihnen hangen auch die jeweilige Ausgestaltung des Informationsfilters, die kognitive Repräsentation des Raumes im Rahmen der individuellen «mental map» sowie die Ausprägung des Filters der Verhaltenssteuerung ab. Gemä $\beta$ dieser Theorie sind im Verhaltensablauf die oben analytisch unterschiedenen Elemente kausal aufeinander bezogen.

Im Vergleich zur Verhaltenstheorie gehen Handlungstheoretiker davon aus, daß menschliche Tätigkeiten, zusätzlich zur Reflexivität, insbesondere durch die Intentionalität bestimmt sind. Jede Handlung ist demgemä $\beta$ von einem Zweck geleitet bzw. auf ein Ziel, eine Intention hin entworfen, um in der Welt eine Veränderung herbeizuführen oder um sie vor einer Veränderung zu bewahren.

Wie aus Abb. 2 hervorgeht, kann man bei jeder Handlung vier Prozeßsequenzen unterscheiden: den Handlungsentwurf, die korrelative Situationsdefinition, die Handlungsverwirklichung sowie die daraus resultierenden beabsichtigten und unbeabsichtigten Handlungsfolgen. Im Handlungsentwurf bezieht sich der Aktor sowohl beim Abwägen zwischen alternativen Zielen als auch bei der Entscheidung für die Mittel zur Erreichung der manifesten Absicht auf ein Referenzmuster der Orientierung. 

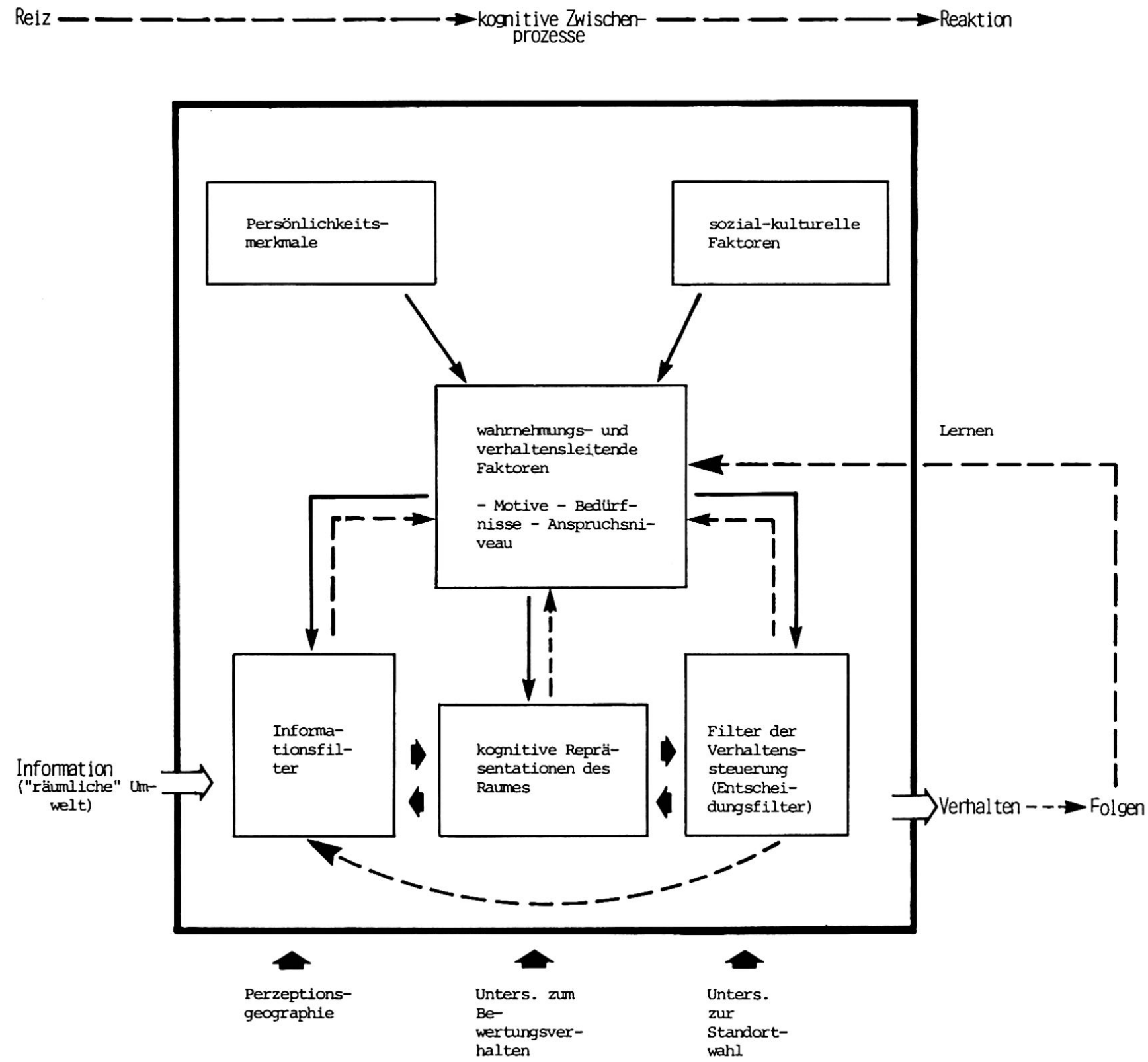

Abb. 1 Verhaltensmodell behavioristischer Sozialgeographie

In der spezifischen Situationsdefinition werden die relevanten und verfügbaren Elemente als Mittel bestimmt und in die Handlungsverwirklichung integriert. Die nicht verfügbaren zielrelevanten Elemente bilden die «Zwänge» des Handelns bzw. der Situation. Jede dieser Sequenzen kann nur verdeckt ablaufen (z. B. ein intellektueller Versuch der Problemlösung) oder auch offenkundig in die äußere Welt gerichtet sein.

Zur Begründung von These 1 sind die folgenden Unterschiede zwischen Verhaltenstheorie und Handlungstheorie als relevante Argumente zu betrachten:

Erstens: Erst über die intentionale Struktur, die sinnhafte Orientierung, wird der gesellschaftliche
Kontext mit seiner historischen Dynamik der Analyse zugänglich gemacht. Denn die logische Struktur der Argumentation der Verhaltenstheorie hat zur Folge, daß soziale Gegebenheiten mit ihren Bedeutungsgehalten als Umwelt, als vorgegebenes Informationsfeld vorausgesetzt werden müssen. Was sie thematisieren kann, sind die Reaktionen des Individuums auf die vorgegebene soziale Welt, nicht aber die Konstitution und absichtsvolle Veränderung der sozialen Wirklichkeit. Da sie die kognitiven Voraussetzungen des Individuums als solche ins Zentrum stellt, aber nicht den Bezug seiner Tätigkeit zu anderen Personen bzw. der sinnhaften Orientierung an deren Aktivitäten, kann man mit ihr nur eine Pycho-Geographie des Individuums, nicht aber 


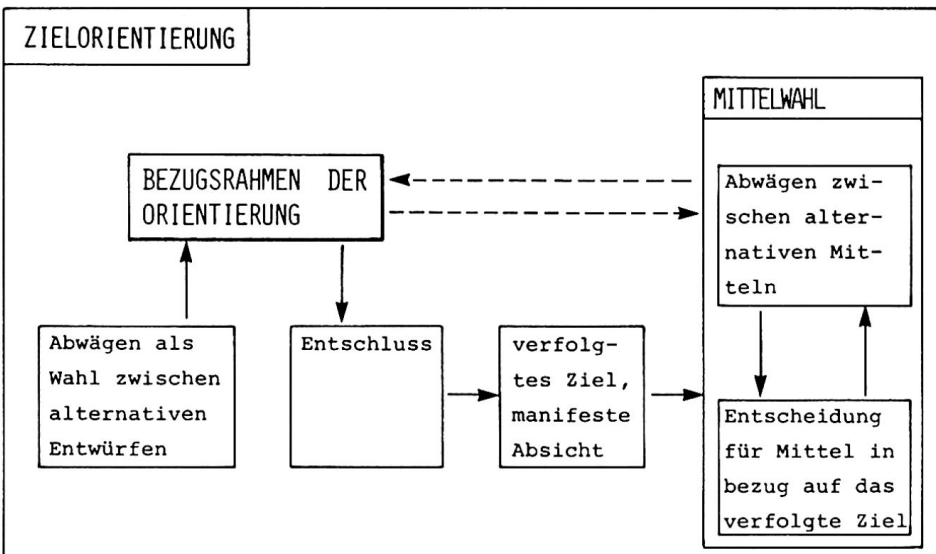

\begin{tabular}{|l|}
\hline HANDELNDER \\
in der \\
Situation I
\end{tabular}

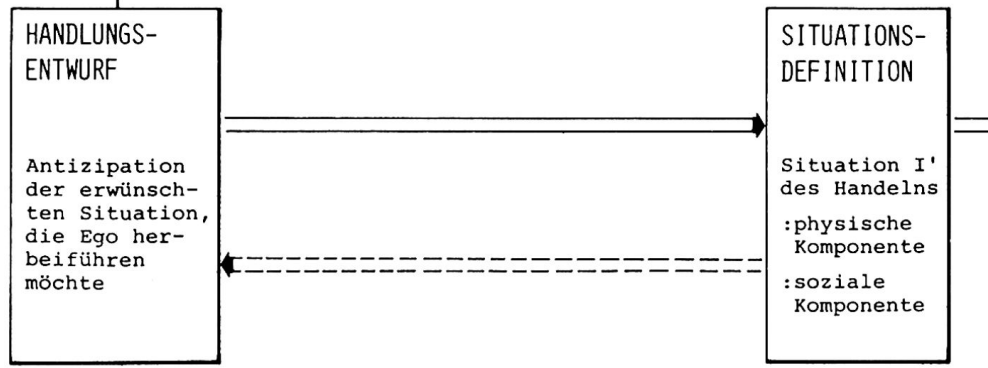

HANDLUNGS-

VERWIRKLI-

CHUNG

gemäss der

Ziel/Zweck-

Mittel Kom-

bination als

Eingriff in

die physische
oder soziale

welt

HANDLUNGS-
FOLGEN
:beabsich-
tigte
oder
:unbeabsich-
tigte Fol-
gen
Situation II

Abb. 2 Modellhafte Rekonstruktion des Handlungsablaufs 
eine Sozial-Geographie begründen. Zweitens: Über die Intentionalität der Handlungen können die bewußten Bezugnahmen auf andere Mitglieder der Gesellschaft thematisiert werden. Beziehen Handlungsentwürfe die Erwartungen eines anderen Aktors in die Erwägungen mit ein, kann man mit WEBER (1980) von sozialen Handlungen im engeren Sinne sprechen. Findet diese Bezugnahme gegenseitig statt, soll man von sozialen Beziehungen sprechen. Treten sie regelmäßig und von gleichen Prinzipien geleitet auf, können sie sich zu verschiedenen Typen sozialer Institutionen wie Markt, Politik usw. ausbilden. Die «Handlung» kann somit als das «Atom» des sozialen Universums betrachtet werden, über das sich die Gesellschaft als primär sinnhafte Wirklichkeit konstituiert und derart in ihrer kleinsten Untersuchungseinheit erforscht werden kann. Drittens: Jeder Versuch, in das Verhaltensmodell Entscheidungen einzuführen, wie dies etwa bei standorttheoretischen Untersuchungen getan wird, ist als inadäquat auszuweisen. Denn jede Entscheidung kann immer nur im Hinblick auf ein bestimmtes Ziel getroffen und somit allein im Rahmen handlungstheoretischer Kategorien widerspruchslos dargestellt werden. Sinnvoller wäre es, für die empirische Forschung angemessenere Modelle des Handelnden zu konstruieren als das stereotype «homooeconomicus»-Modell durch einen verhaltenstheoretisch begründeten «homo irrationalis» zu ersetzen. Damit sei darauf hingewiesen, dass bei logisch konsistenter Übernahme der Verhaltenstheorie bedeutend geringere Anwendungsmöglichkeiten dieses Ansatzes bestehen als dies zur Zeit vorgegeben wird. Eine adäquate handlungstheoretische Neuorientierung der Sozialgeographie eröffnet weit größere und fruchtbarere Forschungshorizonte.

\section{Handlungstheoretische Sozialgeographie der objektiven und der subjektiven Perspektive}

Wenn man der Argumentation zuThese 1 zustimmt, bleibt weiterhin zu klären, auf welchen metatheoretischen Grundlagen eine handlungstheoretische Sozialgeographie aufgebaut werden kann. Die Berücksichtigung erkenntnis- und wissenschaftstheoretischer Forschungsergebnisse soll es erlauben, möglichst viele Paradoxien, wie sie beim «spatial approach» und der humanistischen Alternative bestehen, zu vermeiden. Die drei folgenden Thesen beziehen sich auf diese Absicht.

These 2: Die Metatheorie des Kritischen Rationalismus gibt keine Begründung für die Definition der Sozialgeographie als Raumwissenschaft nach naturwissenschaftlichem Vorbild, sondern als

Handlungswissenschaft der objektiven Perspektive

$a b$.

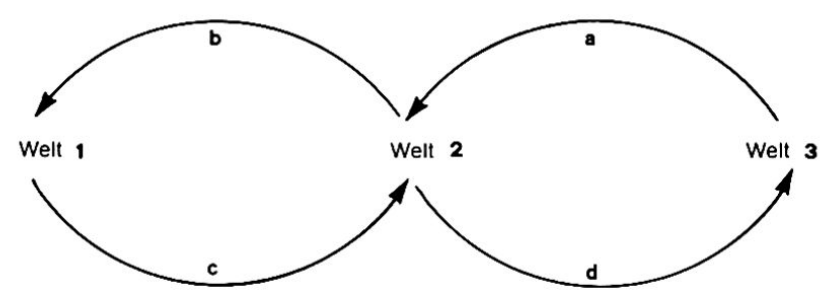

WELT DER PHYSISCHEN GEGENSTÄNDE UND ZUSTÄNDE

physische Seinsweise

WELT DER BEWUSSTSEINSZUSTÄNDE WELT DER IDEEN IM OBJEKTIVEN SINN: DER PROBLEMSITUA TIONEN AN SICH

Abb. 3 Weltbezüge menschlicher Handlungen im Sinne VON POPPER

Wie Abb. 3 zeigt, beruht POPPERs objektive Erkenntnistheorie auf der Unterscheidung von drei «Welten» oder besser: auf der analytischen Untergliederung der Welt in drei ontologisch verschiedene Teilwelten. Als Welt 1 bezeichnet er (vgl. 1973, 123ff.) die physischen Gegenstände und Zustände; als Welt 2 die Gegenstände des Bewußtseins des Menschen; als Welt 3 die Ideen im objektiven Sinne, Problemsituationen an sich und insbesondere die gesellschaftlichen Gegebenheiten (Institutionen, Traditionen usw.). Welt 1 und Welt 3 bestehen nach POPPER objektiv, d. h. unabhängig von der mentalen Welt 2 . Letztere nimmt aber in der Situation des Handelns zwischen den andern beiden eine Vermittlungsfunktion ein: Welt 3 kann nur über das Bewußtsein des Menschen und seine Handlungen aufWelt 1 wirken. Der subjektiven mentalen Welt mißt POPPER in Erkenntnis- und Handlungsprozessen aber keine aktive Rolle bei. Welt 3 ist wohl vom Menschen geschaffen; deren Gegebenheiten, an denen sich der Handelnde orientiert, bleiben aber, einmal fertiggestellt, vom Bewußtsein des Handelnden ebenso unabhängig wie Spinnweben von der Spinne (vgl. 1973, 130).

Die ontologischen Besonderheiten von Welt 1 und Welt 3 weisen wichtige logische Implikationen auf. Die Gegebenheiten der Welt 1 können für andere Gegebenheiten derselben hinreichende Bedingungen sein, Bestandteile der Welt 3 hingegen, unter anderem weil sie Ergebnisse menschlicher Handlungen sind, nur den Status einer notwendigen Bedingung. DieserTatsache müssen auch wissenschaftliche Bezugnahmen auf diese Welten Rechnung tragen: Kausalgesetze und Kausalerklärungen, die nur dann gültig sein können, wenn die «Wenn-Komponente» für die «Dann-Komponente» eine hinrei- 
chende Bedingung darstellt, können nach POPPER $(1967 ; 1969 ; 1980)$ sinnvollerweise nur über die physische Welt, nicht aber über Handlungen und deren Ergebnisse formuliert werden. Für diesen Bereich sollten die Sozialwissenschaftler mittels des Verfahrens der Situationslogik rationale Erklärungen der Handlungen bzw. gesellschaftlicher Gegebenheiten liefern. Da das Verfahren der Situationslogik auf dem Falsifikationsprinzip beruht, widerspricht es nicht dem von POPPER vertretenen Postulat «Einheit der Methode» für alle Wissenschaften.

Diese von POPPER für die Sozialwissenschaften entwickelte Methodologie der Situationslogik kann wie folgt kurz umschrieben werden: Unter den Voraussetzungen, daß Menschen in Handlungssituationen logisch gültig bzw. rational im formalen Sinne vorgehen und daß alle Handlungen vom Ziel der Nutzenmaximierung geleitet sind, «ergibt sich die Möglichkeit, relativ einfache Modelle ihrer Aktionen und Interaktionen zu konstruieren und als Annäherungen zu verwenden» (POPPER, 1969, 110). Wenn diese Annäherungen ausreichend sind, kann man von der objektiven Situation ausgehend die Handlungsabläufe, die auf diese Bezug nehmen, rational erklären. Diese Forschungskonzeption verlangt somit, daß man bei der Gesellschaftsforschung von den objektiven Folgen des Handelns zu den Herstellungsakten fortschreitet, weil die objektive Struktur für die Handlungen als entscheidender betrachtet wird als deren Ziele.

Akzeptiert man POPPERs Argumentation, wird der Vorschlag von BARTELs hinfällig. Da das erdräumliche Gesamtmuster immobiler (materieller) Artefakte denselben logischen Status aufweist wie andere Gegebenheiten, die das Ergebnis sozialer Handlungen bilden, ist die Formulierung empirisch gültiger Kausalgesetze über die erdräumliche Verteilung immobiler (materieller) Artefakte gar nicht möglich. Mein Vorschlag lautet, dieses erdräumliche Gesamtmuster als objektive Struktur der Handlungsfolgen zu betrachten, von dem ausgehend mittels des Verfahrens der Situationslogik eine handlungstheoretische Gesellschaftsforschung in objektiver Perspektive geleistet werden kann.

Wenn man in diesem Zusammenhang davon ausgeht, daß die Verteilungsregelmäßigkeiten immobiler Artefakte, die man mittels der choristisch-chorologischen Methode feststellen kann, das Ergebnis menschlicher Handlungen sind, wird auch fragwürdig, ob die Suche nach «Raumgesetzen» überhaupt sinnvoll ist (3). Es wäre wohl angemessener, diesem erdräumlichen Gesamtmuster nur eine heuristische Funktion zur Aufdeckung von HandlungsregelmäBigkeiten - von "Sinngesetzen», wie sie SOMBART (1930, 253ff.) nennt - zuzuweisen. Ist diese Überlegung zutreffend, kann die Sozialgeographie auch nicht mehr als «handlungsorientierte Raumwissenschaft» (vgl. BARTELS, 1968, 180; 1970, 33) definiert werden. Adäquater wäre dann die Bezeichnung «raumorientierte Handlungswissenschaft».

These 3: Die phänomenologische Metatheorie läßt keine verhaltenstheoretische Grundlegung geographischer Forschung zu, sondern eine handlungstheoretische Forschung der subjektiven Perspektive.

Wie erwähnt, geht POPPER in seiner Wissenschaftsphilosophie von einer «objektiven Erkenntnistheorie ohne erkennendes Subjekt» (POPPER, 1973, 123) aus. Edmund HUSSERL, der Begründer der «Phänomenologie» und Alfred sCHÜTZ, als deren wichtigster Vertreter im Rahmen der Sozialwissenschaften, gehen in erkenntnis- und wissenschaftstheoretischer Hinsicht von einer diametral entgegengesetzten These aus: Die Bedingungen objektiver Erkenntnis sind im erkennenden Subjekt begründet. Es gibt keine subjekt-unabhängige Erkenntnis, denn alles zu Erkennende ist in seinen Bedeutungen vorerst in intentionalen Bewußtseins- und Handlungsakten zu konstituieren.

Auf diesem Hintergrund läßt sich auch bei SCHÜTZ (vgl. 1971, 85ff., 1981, 1982, 110f.) ein Drei-WeltenModell rekonstruieren (vgl. Abb. 4). Er weist darauf hin, daß die physische und die sozial-kulturelle Welt zwar unabhängig vom erkennenden und handelnden Ego besteht, daß aber die Bedeutung ihrer Gegebenheiten immer erst in subjektiven intentionalen Bewußtseinsleistungen konstituiert werden muß. Ausgangspunkt der Erkenntnis ist somit die subjektive Welt, die der primär über Sozialisation vermittelte Wissensvorrat von Ego bildet. Anhand von diesem konstituiert Ego in der Situation des Handelns gemäß scHüTz intentional die Bedeutungen der physischen Welt und die Bedeutungsgehalte der sozial-kulturellen Welt.

Stimmt man der Argumentation von sCHÜTz zu, wird der Anspruch der Vertreter der «humanistic geography», eine verhaltenstheoretische Sozialgeographie konsistent phänomenologisch zu begründen, hinfällig. Denn der verhaltenstheoretische An-

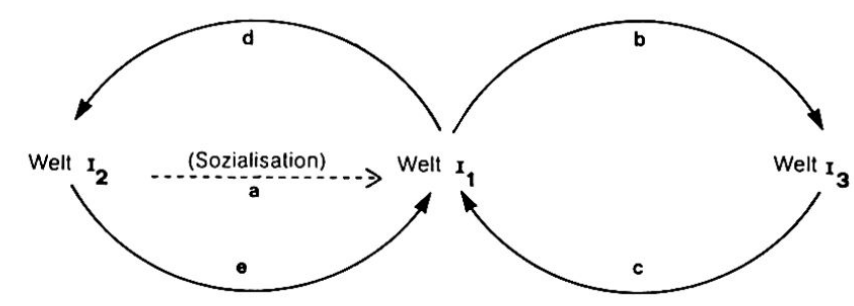

SOZIALE WELT

objektive Sinngehalte, interperso-

SUBJEKTIVE WELT

PHYSISCHE WELT nale Beziehungen

subjektiv verfügbarer Wissensvorrat

Gesamtheit der physischen Dinge

Abb. 4 Weltbezüge menschlicher Handlungen im Sinne von SCHÜTZ 
satz negiert per definitionem die Intentionalität menschlicher Tätigkeiten oder blendet sie zumindest aus, während die phänomenologische Argumentation sie zum Kernstück ihrer Metatheorie macht. Hingegen ist auf dieser Metatheorie in logisch und theoriebegrifflich konsistenter Weise eine Handlungstheorie aufzubauen, denn sie trägt der Intentionalität Rechnung. Und zwar eine Handlungstheorie der subjektiven Perspektive, die von den subjektiven Sinnkonstitutionen der Gegebenheiten der sozialen Welt ausgeht, um diese zu verstehen, teleologisch zu erklären. Darüber hinaus ist auch darauf hinzuweisen, $\mathrm{da} B$ die Rede von «meaning of places" wenig Sinn macht. Orte als solche können keine Sinngehalte aufweisen. Es sind die handelnden Personen, welche den Sinn von Orten konstituieren, und dieser kann von Person zu Person oder besser: von Handlungsintention zu Handlungsintention wechseln. Somit wird auch im phänomenologischen Argumentationskontext deutlich, $\mathrm{da} B$ die sozialgeographische Forschung nicht den «Raum» zu ihrem Forschungsgegenstand erklären kann, sondern sich konsequenterweise auf die Erforschung der Gesellschaft in subjektiver Perspektive ausrichten, d. h. von den subjektiven Sinngehalten der Handlungen ausgehen sollte. In welchem Verhältnis diese Forschungskonzeption zu jener von POPPER stehen kann, ist das Thema der folgenden These:

\section{These 4: Objektive und subjektive Perspektive handlungstheoretischer Gesellschaftsforschung stehen nicht in einem sich gegenseitig ausschließen- den, sondern in einem komplementären Verhältnis.}

Wie wir gesehen haben, geht POPPER von einem absoluten Realismuspostulat aus, d. h. physische wie soziale Wirklichkeit weisen für ihn einen objektiven Status auf, so $\mathrm{da} B$ sie als reale Falsifikationsinstanzen betrachtet werden können. SCHÜTZ hingegen geht von einem relativen Realismuspostulat aus; d. h. jede Realität ist für das handelnde Ego nur in der Form relevant, wie es sie in ihren Bedeutungen konstituiert. Diesen Unterschied zwischen der Argumentation von POPPER und SCHÜTZ soll aber nicht als unüberwindbar betrachtet werden. Denn auch sCHÜTZ leugnet nicht, daß es eine objektive sozialkulturelle Wirklichkeit gibt.

Allerdings setzt er diese nicht als solche naiv voraus, sondern weist nach, da $\beta$ sie das Ergebnis der Erkennitnisleistungen der Subjekte ist, die sie in ihren Bedeutungen auf intersubjektiv gleiche Weise konstituieren. POPPER setzt gemäß dieser Betrachtungsweise die Existenz objektiver sozialer Tatsachen unkritisch voraus und SCHÜTZ fragt danach, unter welchen Bedinungungen sie entstehen und bestehen können.

Wenn ein Sozialwissenschaftler ein soziales Problem lösen möchte, dann ist es nach SCHÜTZ notwendig, zuerst zu wissen, in welchen subjektiven Sinnzusammenhängen die Handlungen gestanden haben, die dazu geführt haben. Daraus schließe ich, da $B$ in jenen Fällen, in denen der Sozialwissenschaftler sicher sein kann, daß die Handlungen in einem intersubjektiv bekannten bzw. quasi objektiven Sinnzusammenhang stehen, er nicht die subjektive Perspektive einzunehmen braucht. Er kann dann gemäß der POPPERschen Konzeption vorgehen, die mit wesentlich geringerem Forschungsaufwand verbunden ist.

Beide Forschungsperspektiven können somit in einem sich ergänzenden Verhältnis gesehen werden. Dies um so eher, als POPPER und sCHÜTZ die Postulate des methodologischen Individualismus und der Wertneutralität, die Einschätzung der Bedeutung von Modellen für die Sozialforschung und die Prinzipien der Modellbildung teilen.

\section{Sozialwissenschaftliche Leitlinien}

Selbst wenn die bisherigen Überlegungen für die Abstützung der neu zu entwickelnden sozialgeographischen Forschungspraxis erste Orientierungshilfen abgeben können, ist aber noch nicht entschieden, auf welche sozialwissenschaftlichen Handlungstheorien dafür zurückzugreifen ist. Jürgen HA. BERMAS (1981) vertritt die Auffassung, da $\beta$ die bisherigen Handlungstheorien alle nur einen verkürzten Forschungszugang zur Gesellschaft ermöglichen und $\mathrm{da} B$ sie durch die von ihm entworfene "Theorie des kommunikativen Handelns» zu ersetzen wären. Diesem Vorschlag stelle ich die folgende These gegenüber:

\section{These 5: Zweckrationale, norm- und verständi- gungsorientierte Handlungstheorien eröffnen zunehmend umfassendere Zugänge zur sozialen Wirklichkeit, auf die die sozialgeographische Forschung, je nach dem zu lösenden Problem, zurückgreifen soll.}

Die Klärung der sozialwissenschaftlichen Grundlagen handlungstheoretischer Sozialgeographie verlangt zuerst nach einer Ordnung der verschiedenen Handlungstheorien, um sie erstens vergleichbar zu machen und zweitens eine kontrollierte Bezugnahme zu ermöglichen. Als primäres Ordnungskriterium bietet sich das bei der Erläuterung von These 4 angesprochene $\mathrm{MaB}$ der vorgenommenen Einklammerung sozialer Wirklichkeit an. Diesbezüglich kann zwischen zweckrationalen, norm- und verständigungsorientierten Handlungstheorien unterschieden werden. Unter die erste Gruppe werden die Theorie der logischen Handlungen von v. PARETO (1917), jene der zweckrationalen Handlungen von M. WEBER $(1951,1980)$ und die Entscheidungstheorie (vgl. GAEFGEN, 1974) subsumiert. Als norm- 
orientierte Ansätze werden die struktur-funktionale Theorie von T. PARSONS (1937, 1952) und die Theorien der übrigen funktionalistischen Gesellschaftstheoretiker gezählt. $\mathrm{Zu}$ den verständigungsorientierten Basiskonzepten sozialwissenschaftlicher Forschung gehört die von SCHÜTZ (1971, 1972, 1979, 1984) entwickelte Grundlegung der Soziologie und die darauf aufbauende Ethnomethodologie.

Für den differenzierten Vergleich und eine streng kontrollierbare Bezugnahme reicht dies noch nicht. Darüber hinaus können in struktureller Hinsicht die in Abb. 2 unterschiedenen Kategorien verwendet werden: das vorausgesetzte Modell des Handelnden, die Zielorientierung des Handelns, den jeweils thematisierten Bezugsrahmen der Orientierung und die rekonstruktive Darstellung der Situation des Handelns. Es würde hier zu weit führen, jede einzelneTheorie hinsichtlich dieser Kategorien ausführlich zu charakterisieren. Hingegen sollen die Verwendungsmöglichkeiten präzisiert werden:

Auf jedem der angeführten Ansätze kann die handlungstheoretische Neuorientierung der Sozialgeographie aufbauen. Welcher in der Forschungspraxis, unter Berücksichtigung der im Folgenden noch zu besprechenden Erweiterung, zur Anwendung kommt, soll von der Art des zu lösenden oder zu rekonstruierenden Problems oder den theoretischen Interessen abhängen:

- Bildet die optimale Mittelwahl bei akzeptierten Zielen das Problem, d. h. geht es um die Lösung oder die Erforschung technischer Aspekte, dann ist auf die Gruppe der zweckrationalen Handlungstheorien Bezug zu nehmen (z. B. Standortwahl als Mittel der Gewinnmaximierung).

- Sind soziale Normen und kulturelle Werte der Ziel- und/oder Mittelwahl zu rekonstruieren sowie problematische Aspekte der sozialen Ordnung zu erforschen, ist auf die normorientierten Handlungstheorien zurückzugreifen.

- Ist das wissenschaftliche oder praktische Problem mit der Klärung umfassender Verständigungsdivergenzen, d.h. mit divergierenden (subjektiven) Sinnkonstitutionen verbunden, dann sollte die Forschung gemäß den verständigungsorientierten Theorien betrieben werden.

Die damit vorgeschlagene Vorgehensweise ist von dem Prinzip der «Forschungsökonomie» geleitet. Denn je umfassender die soziale Wirklichkeit thematisiert wird, desto größer fällt auch der damit verbundene empirische Forschungsaufwand aus. Deshalb sollten im ersten Zugang nur jene Bereiche erfaßt werden, die hypothesenrelevant sind. Wenn sich aber zeigt, daß bestimmte Probleme, wie die wirtschaftliche Unterentwicklung der Dritten Welt, den Zuständigkeitsbereich der zweckrationalen Theorie (der Ökonomie) übersteigt, dann muß eine Lösung in umfassenderen Theoriehorizonten angestrebt werden.

\section{Sozialgeographie als raumorientierte Handlungs- wissenschaft}

Wenn davon ausgegangen wird, daß jede legitimierbare wissenschaftliche Disziplin einen besonderen Beitrag zur Lösung lebenspraktischer Probleme liefern sollte, dann müßte eine handlungstheoretische Sozialgeographie in der Lage sein, die sozialwissenschaftlichen Handlungstheorien in bestimmter Hinsicht zu spezifizieren. Neben deren Anwendung auf besondere Themenbereiche bedeutet dies im Hinblick auf das Forschungsziel der Sozialgeographie, daß es gelingen muß, die bisherigen formalen Kategorien durch eine zusätzliche - die räumliche Dimension - zu erweitern und dementsprechend ihren Forschungsgegenstand neu $\mathrm{zu}$ definieren. So schlage ich vor, die «Analyse menschlicher Handlungen in ihren räumlichen Bezügen» zum Basisbereich handlungstheoretischer Sozialgeographie zu machen. Die letzte These betrifft einen damit zusammenhängenden Problembereich:

\section{These 6: Soll die sozialgeographische Gesellschafts- forschung nicht an inadäquaten Reduktionen scheitern, so ist, im Sinne einer Vorbedingung, für jeden ontologisch differenten Bezugsbereich menschlicher Handlungen ein besonderes Referenz- muster der Orientierung und Lokalisierung verfügbar zu machen.}

Bevor eine wissenschaftliche Beschreibung und Erklärung geleistet werden kann, sind zuerst die problematischen und/oder relevanten Gegebenheiten zu strukturieren und zu lokalisieren. Dafür müssen formale Ordnungsraster verfügbar gemacht werden. Will man dabei nicht an inadäquaten Reduktionen scheitern, ist im Hinblick auf den hier vorgeschlagenen sozialgeographischen Forschungsgegenstand für jeden Bezugsbereich menschlicher Handlungen ein besonderes Referenzmuster zu entwikkeln. Diese Referenzmuster sollen sowohl zur Rekonstruktion der Handlungsorientierungen in der sozialen, mentalen und sozial-kulturellen Welt Verwendung finden können, um Handlungsabläufe unverkürzt erklären zu können, als auch - im Rahmen von Strukturanalysen - der Lokalisierung von handlungsrelevanten Gegebenheiten dienen. Mein Vorschlag lautet, da $\beta$ derartige Referenzmuster als Raumbegriffe der objektiven und der subjektiven Perspektive thematisiert werden sollen, deren Merkmalsdimensionen mit dem ontologischen Status der zu lokalisierenden Gegebenheit verträglich sind.

Die Bedeutung dieser Forderung wird dann offensichtlich, wenn man sich die bisher erfolglosen Versuche der Sozialgeographen vor Augen führt, soziale Sinngehalte in der physischen Welt eindeutig zu lokalisieren. Die Aufforderung beispielsweise, 
daß Sozialgeographen Ausschnitte der Erdoberfläche abgrenzen sollen, innerhalb denen gleiches «sozialgeographisches Verhalten» auftrete, wurde bereits in den vierziger Jahren von BOBEK vorgebracht und dann von HARTKE, den Münchener Sozialgeographen bis zu BARTELS - wenn auch in unterschiedlichen Formulierungen - wiederholt. Keiner empirischen Untersuchung ist das aber je gelungen. Nach der hier vertretenen Auffassung ist dies auch unmöglich, weil die sozial-kulturellen Gegebenheiten, wie Normen, Werte u. a. keinen materiellen Status aufweisen und somit im Erdraum auch nicht eindeutig lokalisiert werden können. Denn im Erdraum nimmt «nur» der Körper des Handelnden einen eindeutig definierbaren Standort ein, nicht aber die Sinngehalte, die seine Tätigkeiten leiten. Jeder Versuch, die Bestimmungsgründe des Handelns im Erdraum zu fixieren, kommt einer inadäquaten Reduktion des Sozial-Kulturellen und seinen subjektiven Interpretationen (POPPER) bzw. Grundlagen (sCHÜTZ) gleich, die jede empirisch gültige Aussage unmöglich machen. Genau deshalb brauchen wir für jeden ontologisch differenten $\mathrm{Be}$ reich ein besonderes Referenzmuster der Lokalisierung. Ich möchte diesen Gedankengang anhand der Metatheorie von POPPER präzisieren.

In objektiver Forschungsperspektive stehen gemä $B$ POPPER Welt 1 und Welt 3 im Vordergrund. Für die physische Welt, d.h. für alle materiellen Dinge ohne sozialen Sinngehalt, reicht zur eindeutigen Lokalisierung der aus dem mechanisch-euklidischen Raumbegriff abgeleitete traditionelle chorische Raumbegriff der Geographie aus. Die Dimensionen «Länge» und «Breite» bzw. «Längen»- und «Breitengrade», der beliebig wählbare «Koordinatennullpunkt», die "Himmelsrichtungen» und ein «metrisches Einheitsmaß» definieren diesen «metrischen Ordnungsrahmen eines erdräumlichen Kontinuums» (BARTELS, 1974, 13). Anhand von ihm können Standortmuster und Bewegungen von materiellen Dingen ohne Sinngehalt und von materiellen Artefakten hinsichtlich ihrer physischen Komponente beschrieben und strukturiert werden.

Für die Entwicklung eines formalen Referenzschemas zur Lokalisierung sozial-kultureller Sinngehalte hat SOROKIN (1964) die ersten Vorarbeiten geleistet, die Sozialgeographen weiterentwickeln sollten. Als Hauptdimensionen, auf denen sozial-kulturelle Sinngehalte lokalisierbar werden, welche die Handlungen leiten und/oder in Artefakten aufgehoben `sind, schlägt er «Sprache», «Kunst», «Ethik» (=Moral und Recht), "Wissenschaft», «Religion» sowie «Wirtschaft» und "Politik» vor, außerdem feinere Abstufungen jeder dieser Merkmalsdimensionen. Der Koordinatennullpunkt, auf den sich die verschiedenen Dimensionen beziehen, ist nach soROKIN je nach dem Interesse der Untersuchung ebenso beliebig wählbar wie beim Ordnungsschema des erdräumlichen Kontinuums.
Für die subjektive Forschungsperspektive eröffnet sich, unter Berücksichtigung der Grundprinzipien von SCHÜTZ, eine vergleichbare Erweiterungsmöglichkeit, auf die ich hier aber nicht ausführlich eingehen möchte. Jedenfalls wäre davon auszugehen, daß der Erkennende und Handelnde seine Mitwelt subjektbezogen erfährt, so daß er die physische Welt in bezug auf seinen Leib und die Sinndimensionen der sozial-kulturellen Welt in bezug auf die biographische Ausprägung des subjektiv verfügbaren Wissensvorrates strukturiert und sich dementsprechend in diesen Welten orientiert. Die Entwicklung ontologisch adäquater Referenzschemata müßte diesen Zusammenhängen Rechnung tragen, wenn die sozialgeographischen Forschungen in subjektiver Perspektive empirische Gültigkeit beanspruchen wollen.

\section{Schlußbemerkung}

Das Hauptanliegen dieser Ausführungen besteht darin, die Voraussetzungen des Programms «Handlungstheoretische Sozialgeographie» zu klären, die einzelnen Aspekte anhand von sechs Thesen und deren Erläuterung zu präzisieren sowie Orientierungsrichtlinien für die forschungspraktische Umsetzung vorzuschlagen. Nicht berücksichtigt wurden hier u. a. die thematischen und formalen Anknüpfungspunkte, die sich für die handlungstheoretische Neuorientierung im Rahmen der Sozialwissenschaften und der bisherigen sozialgeographischen Forschung anbieten, welche die Verwirklichung des vorgeschlagenen Vorhabens in nicht allzuferner $\mathrm{Zu}$ kunft erleichtern sollten.

\section{Anmerkungen}

1 Im folgenden Text möchte ich die Hauptthesen meiner Dissertation (WERLEN, 1986) und die entsprechende Argumentation vorstellen, bei der hier aber wichtige Sonderprobleme, aus wohl verständlichen Gründen, unberücksichtigt bleiben müssen.

2 Die späteren Vorschläge von E. WIRTH (1977; 1979; 1980; 1981) können dieselben und andere Mängel nicht überwinden und die Konzeption von P. SEDLACEK (1982) bleibt auf einen engen Themenbereich beschränkt (vgl. WERLEN, 1986, 405-415).

3 Die Sinnhaftigkeit der Suche nach "Raumgesetzen" wird von den Vertretern des raumwissenschaftlichen Ansatzes entweder nicht gestellt oder eindeutig positiv beantwortet. Dabei kann aber eine Inkonsistenz ihrer Argumentation nicht verborgen bleiben. Einerseits wird davon ausgegangen (vgl. BARTELS, 1974), daß mit der Lokalisierung einer Gegebenheit im erdräumlichen Kontinuum nur ein formaler Aspekt von ihr festgehalten wird. So müßte man annehmen können, daß auch die Distanz zwischen zwei Standorten nur formale Merkmale zum Ausdruck bringt. Trotzdem werden aber Distanzvariablen als produktive Ursachen für das Auftreten und die Verteilung von Sachverhalten betrachtet (vgl. BAR- 
TELS, 1970). Dies kommt einer inadäquaten Reifikation gleich. Lehnt man sie ab, kann man auch nicht mehr die Aufdeckung kausaler "Gesetzmäßigkeiten in den distanziellen Lagebeziehungen von Beobachtungseinheiten aller Art" (BARTELS 1974, 15) als Zielsetzung der Sozialgeographie akzeptieren.

\section{Literatur}

BARTELS, D. (1968): Zur wissenschaftstheoretischen Grundlegung einer Geographie des Menschen. Wiesbaden. BARTELS, D. (1970): Einleitung. In: BARTELS, D. (Hrsg.) Wirtschafts- und Sozialgeographie. Köln/Berlin, S. 13-48.

BARTELS, D. (1973): Between theory and metatheory. In: CHORLEY R. J. (ed.) Directions in geography. London, S. 23-42.

BARTELS, D. (1974): Schwierigkeiten mit dem Raumbegriff in der Geographie. In: Beihefte zu Geographica Helvetica 29, Nr. 2/3, S. 7-21.

BARTELS, D., HARD, G. (1975): Lotsenbuch für das Studium der Geographie als Lehrfach. 2. Aufl. Bonn/Kiel.

BUNGE, W. (1966): Theoretical Geography. Lund.

BUTTIMER, A. (1976): Grasping the dynamism of Lifeworld. In: Annals of the Association of American Geographers, 66, S. 277-297.

BUTTIMER, A. (1979): Le temps, l'espace et le monde vécu. In: L'Espace géographique, S. 243-254.

CLAVAL, P. (1970): Geographie als sozialwissenschaftliche Disziplin. In: BARTELS, D. (Hrsg.): Wirtschafts- und Sozialgeographie. Köln/Berlin. S. 418-434.

GÄFGEN, G. (1974): Theorie der wirtschaftlichen Entscheidung. 3. Aufl. Tübingen.

GOLD, J. R. (1980): An Introduction to Behavioural Geography. Oxford.

HABERMAS, J. (1981): Theorie des kommunikativen Handelns. 2 Bd. Frankfurt a. M.

LEY, D. (1979): Social geography and the taken-for-grantedworld. In: GALE, ST./OLSSON, G. (eds.): Philosophy in Geography. Dodrecht/Boston/London. S. 215-236.

LEY, D. (1981): Cultural/humanistic geography. In: Progress in Human Geography. 5, S. 251-257.

LEY, D. (1985): Cultural/humannistic geography. In: Progress in Human Geography. 9, S. 415-423.

PARETO, V. (1917): Traité de sociologie générale. Paris.

PARSONS, T. (1937): Structures of Social Action. Glencoe III.

PARSONS, T. (1952): The Social System. London.

POPPER, K. R. (1967): La rationalité et le status du principe de rationalité. In: CLASSEN, E. M. (éd.): Les fondements philosophiques des systèmes économiques, Paris, S. 142-150.
POPPER, K. R. (1969): Das Elend des Historizismus. Tübingen.

POPPER, K. R. (1973): Objektive Erkenntnis. Ein evolutionärer Entwurf. Hamburg.

POPPER, K.R. (1980): Die offene Gesellschaft und ihre Feinde. Bd. 2, 6. Aufl. München.

SCHÜTZ, A. (1971): Gesammelte Aufsätze. Bd. 1: Das Problem der sozialen Wirklichkeit. Den Haag.

SCHÜTZ, A. (1972): Gesammelte Aufsätze. Bd. 2: Studien zur soziologischen Theorie. Den Haag.

SCHÜTZ, A. (1981): Theorie der Lebensformen. Frankfurt a. M.

SCHÜTZ, A. (1982): Das Problem der Relevanz. Frankfurt a. M.

SCHÜTZ, A., LUCKMANN, TH. (1979): Strukturen der Lebenswelt. Bd. 1, Frankfurt a. M.

SCHÜTZ, A., LUCKMANN, TH. (1984): Strukturen der Lebenswelt. Bd. 2, Frankfurt a. M.

SEDLACEK, P. (1982): Kulturgeographie als normative Handlungswissenschaft. In: SEDLACEK, P. (Hrsg.) Kultur-/Sozialgeographie. Paderborn. S. 187-216.

SOMBART, W. (1930): Die drei Nationalökonomien. München/Leipzig.

SOROKIN, P.A. (1964): Sociocultural Causality, Space, Time. New York.

TUAN, Y. F. (1976): Humanistic Geography. In: Annals of the Association of American Geographers, 66, S. 266-276.

WEBER, M. (1951): Gesammelte Aufsätze zur Wissenschaftslehre. Tübingen.

WEBER, M. (1980): Wirtschaft und Gesellschaft. 5. Aufl. Tübingen.

WERLEN, B. (1983): Methodologische Probleme handlungstheoretischer Stadtforschung. In: LÖTSCHER, L. (Hrsg.) Stadtdynamik in der Schweiz. Jahrbuch der Schweizerischen Naturforschenden Gesellschaft, wissenschaftlicher Teil, 1982, Bd. 2, S. 100-109.

WERLEN, B. (1986): Handlungstheoretische Sozialgeographie. Eine Untersuchung der metatheoretischen und sozialwissenschaftlichen Grundlagen sowie der Ausgangsbedingungen in der deutschen Sozialgeographie (unveröffentl. Dissertation). Fribourg.

WIRTH, E. (1977): Die deutsche Sozialgeographie in ihrer theoretischen Konzeption und in ihrem Verhältnis zu Soziologie und Geographie des Menschen. In: Geographische Zeitschrift, 65, S. 161-187.

WIRTH, E. (1979): Theoretische Geographie. Stuttgart

WIRTH, E. (1980): Zwei Jahrzehnte Theoretische Geographie. Eine kritische Bilanz. In: Dt. Geographentag 1979 Göttingen. Wiesbaden. S. 167-179.

WIRTH, E. (1981): Kritische Anmerkungen zu den wahrnehmungszentrierten Forschungsansätzen in der Geographie. In: Geographische Zeitschrift, 69, S. 161-198. 